\title{
1 Precision Metabolic Engineering: the Design of Responsive, Selective, 2 and Controllable Metabolic Systems
}

4 Monica P. McNerney, Daniel M. Watstein, and Mark P. Styczynski* 5

6 School of Chemical \& Biomolecular Engineering, Georgia Institute of Technology, Atlanta, GA, 7 USA

9 *Corresponding author. Address: 311 Ferst Drive NW, Atlanta, GA 30332-0100, USA; email:

10 Mark.Styczynski@ chbe.gatech.edu

11

12 Keywords

13 Precision metabolic engineering; metabolic control; sensory systems; pathway regulation; product 14 selectivity; synthetic biology

15

16 


\section{Abstract}

18 Metabolic engineering is generally focused on static optimization of cells to maximize

19 production of a desired product, though recently dynamic metabolic engineering has explored how

20 metabolic programs can be varied over time to improve titer. However, these are not the only types

21 of applications where metabolic engineering could make a significant impact. Here, we discuss a

22 new conceptual framework, termed "precision metabolic engineering," involving the design and

23 engineering of systems that make different products in response to different signals. Rather than

24 focusing on maximizing titer, these types of applications typically have three hallmarks: sensing

25 signals that determine the desired metabolic target, completely directing metabolic flux in response

26 to those signals, and producing sharp responses at specific signal thresholds. In this review, we

27 will first discuss and provide examples of precision metabolic engineering. We will then discuss

28 each of these hallmarks and identify which existing metabolic engineering methods can be applied

29 to accomplish those tasks, as well as some of their shortcomings. Ultimately, precise control of

30 metabolic systems has the potential to enable a host of new metabolic engineering and synthetic

31 biology applications for any problem where flexibility of response to an external signal could be 32 useful. 


\section{Introduction}

Metabolic engineering efforts typically focus on designing an organism to maximize the final yield of a desired product, as this is usually the most commercially important goal. This approach has been successfully used to create and improve microbial production of high-demand products such as biofuels (Atsumi et al., 2008a, 2008b; Jin et al., 2005), pharmaceuticals (AlonsoGutierrez et al., 2013; Ro et al., 2006), and commodity chemicals (Chemler et al., 2010; Raab et al., 2010). However, there are a number of applications where it is more important to design systems that can flexibly respond to different signals and tightly control output levels, rather than just produce as much of one single compound as possible. This class of problems can be referred to as "precision metabolic engineering", and it is an area that will likely receive greater attention as metabolic engineering finds more diverse applications.

The fundamental goals and characteristics of precision metabolic engineering are the necessity for a singular output determined by the state of the system and an emphasis on maximizing product selectivity rather than just final titer. Based on the flexibility required for this approach, it is in some ways similar to the growing field of dynamic metabolic engineering (Brockman and Prather, 2015a; Venayak et al., 2015), in which metabolic flux is redirected as the system changes during the production process. The main contrast is that while the hallmark of dynamic metabolic engineering is control of metabolism as a function of time to maximize titer and productivity, precision metabolic engineering instead emphasizes having metabolic states that are completely and sharply switchable in response to a specific input (though temporal control could play a role in some precision metabolic engineering applications). While their objectives differ, many techniques used for dynamic control of metabolism can be used to implement precise control over metabolic systems. Precision metabolic engineering is relevant in any situation where a portable microbial cell factory capable of responding to external signals with multiple metabolic outputs could be useful.

\begin{tabular}{|l|l|l|l|}
\hline Characteristic & $\begin{array}{l}\text { General Metabolic } \\
\text { Engineering }\end{array}$ & $\begin{array}{l}\text { Dynamic Metabolic } \\
\text { Engineering }\end{array}$ & $\begin{array}{l}\text { Precision Metabolic } \\
\text { Engineering }\end{array}$ \\
\hline $\begin{array}{l}\text { Engineering } \\
\text { optimization } \\
\text { goal }\end{array}$ & $\begin{array}{l}\text { Product titer, yield, } \\
\text { productivity }\end{array}$ & $\begin{array}{l}\text { Product titer, yield, } \\
\text { productivity }\end{array}$ & $\begin{array}{l}\text { State-based selectivity and } \\
\text { response sensitivity }\end{array}$ \\
\hline
\end{tabular}




\begin{tabular}{|c|c|c|c|}
\hline $\begin{array}{l}\text { Temporal } \\
\text { behavior }\end{array}$ & $\begin{array}{l}\text { None; optimization } \\
\text { through static } \\
\text { manipulations }\end{array}$ & $\begin{array}{l}\text { System behavior } \\
\text { necessarily changes } \\
\text { over time }\end{array}$ & $\begin{array}{l}\text { System behavior may (but } \\
\text { does not necessarily) } \\
\text { change over time }\end{array}$ \\
\hline $\begin{array}{l}\text { Number of } \\
\text { possible states }\end{array}$ & $\begin{array}{l}\text { One; static optimization } \\
\text { produces singular } \\
\text { desired state }\end{array}$ & $\begin{array}{l}\text { Two; change } \\
\text { between states } \\
\text { initiated at time } \\
\text { point during } \\
\text { production } \\
\end{array}$ & $\begin{array}{l}\text { Many; states could change } \\
\text { over time or be } \\
\text { determined by initial } \\
\text { concentration of sensory } \\
\text { molecule }\end{array}$ \\
\hline $\begin{array}{l}\text { Signal that } \\
\text { dictates } \\
\text { metabolic } \\
\text { state }\end{array}$ & $\begin{array}{l}\text { None; state of system } \\
\text { predetermined }\end{array}$ & $\begin{array}{l}\text { Exogenous } \\
\text { molecules, naturally } \\
\text { produced } \\
\text { metabolites }\end{array}$ & $\begin{array}{l}\text { Exogenous molecules, } \\
\text { naturally produced } \\
\text { metabolites }\end{array}$ \\
\hline $\begin{array}{l}\text { Methods used } \\
\text { to change } \\
\text { metabolic } \\
\text { state }\end{array}$ & $\begin{array}{l}\text { None; state of system } \\
\text { predetermined }\end{array}$ & $\begin{array}{l}\text { Inducible } \\
\text { transcriptional, post- } \\
\text { transcriptional } \\
\text { regulation }\end{array}$ & $\begin{array}{l}\text { Inducible transcriptional, } \\
\text { post-transcriptional } \\
\text { regulation }\end{array}$ \\
\hline $\begin{array}{l}\text { Necessary } \\
\text { degree of } \\
\text { control } \\
\text { between } \\
\text { multiple } \\
\text { desired } \\
\text { metabolic } \\
\text { states }\end{array}$ & $\begin{array}{l}\text { None; state of system } \\
\text { predetermined }\end{array}$ & $\begin{array}{l}\text { Majority of } \\
\text { metabolic flux must } \\
\text { be through desired } \\
\text { pathways, but } \\
\text { leakiness in } \\
\text { transcription and } \\
\text { enzymatic activity } \\
\text { may be permitted }\end{array}$ & $\begin{array}{l}\text { All measurable metabolic } \\
\text { flux must be through } \\
\text { desired pathways, } \\
\text { leakiness in transcription } \\
\text { and enzymatic activity not } \\
\text { permitted }\end{array}$ \\
\hline Examples & $\begin{array}{l}\text { Industrial production of } \\
\text { biofuels, } \\
\text { pharmaceuticals, } \\
\text { commodity chemicals }\end{array}$ & $\begin{array}{l}\text { Industrial production } \\
\text { in which titer and } \\
\text { productivity can be } \\
\text { increased either by } \\
\text { shifting from a } \\
\text { growth phase to a } \\
\text { production phase or } \\
\text { by balancing fluxes } \\
\text { to reduce toxic } \\
\text { intermediates }\end{array}$ & $\begin{array}{l}\text { Metabolite biosensors, } \\
\text { portable pharmaceutical } \\
\text { production, targeted drug } \\
\text { delivery }\end{array}$ \\
\hline
\end{tabular}

Table 1: Comparison of precision metabolic engineering, dynamic metabolic engineering, and general metabolic engineering approaches

One example of where precise control could be valuable is in the development of bacterial

63 biosensors. Bacterial biosensors have been designed to sense and respond to the presence of

64 harmful chemicals (Cheol Gil et al., 2000), radiation (Rosen et al., 2000), and heavy metals (Verma

65 and Singh, 2005), though they often use fluorescent reporters or reporters that require addition of 66 exogenous substrate for enzymatic reactions (e.g., luminescence), which limits utility in low-

67 resource (electricity and equipment) environments. Precision metabolic engineering using only 
sugar substrates can be used to produce metabolite outputs (such as pigments) that are visible without the use of equipment, thus enabling such biosensors to be more widely used in lowresource environments. Extensive metabolic engineering efforts have already been made to increase microbial production of pigmented metabolites such as lycopene (Alper et al., 2005a;

72 Farmer and Liao, 2000; Yoon et al., 2006) and violacein (Fang et al., 2015; Lee et al., 2013;

73 Rodrigues et al., 2013). By tightly controlling conditions under which these metabolites are

74 produced, they could be used as indicators for biosensors for diverse applications. One such 75 application is biomedical: the development of a biosensor for a blood test to detect micronutrient deficiencies, which are most prevalent in low-resource areas and thus could gain substantially from having essentially equipment-free approaches for diagnosis. Recently our group (Watstein et al., 2015) reported engineered bacteria that produce visible pigments in response to different levels of zinc (an important mineral in human diet), which would enable their use as low-cost, point-of-care assays to detect micronutrient levels. In contrast to traditional metabolic engineering goals, the pigment-based biosensor was engineered not to maximize titer of the final product, but to instead maximize selectivity of pigment production based on the concentration of zinc, subject to the constraint of visible pigment production in a reasonable amount of time - a challenge of precision metabolic engineering. This whole-cell biosensor framework could also be extended and used to detect levels of other micronutrients or blood components.

Applications of precision metabolic engineering extend beyond diagnostics to pharmaceutical production. While pharmaceuticals are generally produced in tightly controlled industrial settings, there is a potentially significant value for flexible "on demand" drug production in certain situations. For example, because of the cost and logistical challenges of storing pharmaceuticals and protein therapeutics for portable use (e.g., by warfighters), DARPA established a battlefield medicine program to develop systems that can produce them in response to situations as they arise. Cells already designed to make pharmaceuticals could be further engineered to produce different therapeutics based on battlefield needs. Importantly, precision

94 metabolic engineering would be needed to ensure that only the desired substance is produced in a certain condition to maximize product purity in the absence of industrial separation processes. Since the program's initiation in 2012, four different drugs have met DARPA's standards of being

97 producible on demand (Choi and Ling, 2014), and incorporating selectivity and control into existing metabolic engineering techniques could contribute to the program's success. 
In addition to producing pharmaceuticals, microbes could also serve as specific drug

100 delivery vehicles. The bacterium Salmonella typhimurium has been explored as novel anticancer

101 vector (Pawelek et al., 1997) because of its protection from immune system clearance and its

102 predilection for the hypoxic environment of a tumor (Brown and Wilson, 2004). Bacteria can be

103 programmed to release cytotoxic agents, cytokines, or tumor antigens in the presence of the tumor,

104 but suboptimal targeting efficiency and intrinsic bacterial toxicity have limited their use as clinical

105 therapeutics (Forbes, 2010). Increasing metabolic control could help combat these challenges: cells

106 could be engineered to release anti-cancer drugs only in the presence of cancer signaling

107 molecules, and a population control mechanism could trigger a kill switch if bacteria accumulate

108 in organs at dangerously high levels.

109 In this review, we will discuss the characteristics of precision metabolic engineering and 110 ways that existing metabolic engineering techniques can be used in applications that require 111 precise metabolic control. For metabolic engineering to be used in situations where tight control 112 is necessary, a system must have the machinery to sense signals that determine the desired 113 metabolic target, must completely direct metabolic flux based on those signals, and must produce 114 sharp responses based on specific signal thresholds (Figure 1). First, we will describe sensory 115 systems that can respond to autonomous and exogenous signals and the ways that protein 116 engineering can enhance selectivity and sensitivity of a sensor. Next, we will describe the ways 117 that different types of transcriptional and post-transcriptional regulation can be used, often in 118 combination, to direct metabolic flux to only the desired pathway. Finally, we will describe 119 methods that can be used to make the system respond sharply to the signal thresholds dictated by 120 the application. Since dynamic metabolic engineering and precision metabolic engineering do have 121 a bit in common and much more work has been done in dynamic than precision metabolic 122 engineering, we will often first refer to applications of specific techniques in a dynamic metabolic 123 engineering context before evaluating their relevance for direct application to precision metabolic 124 engineering. 
126

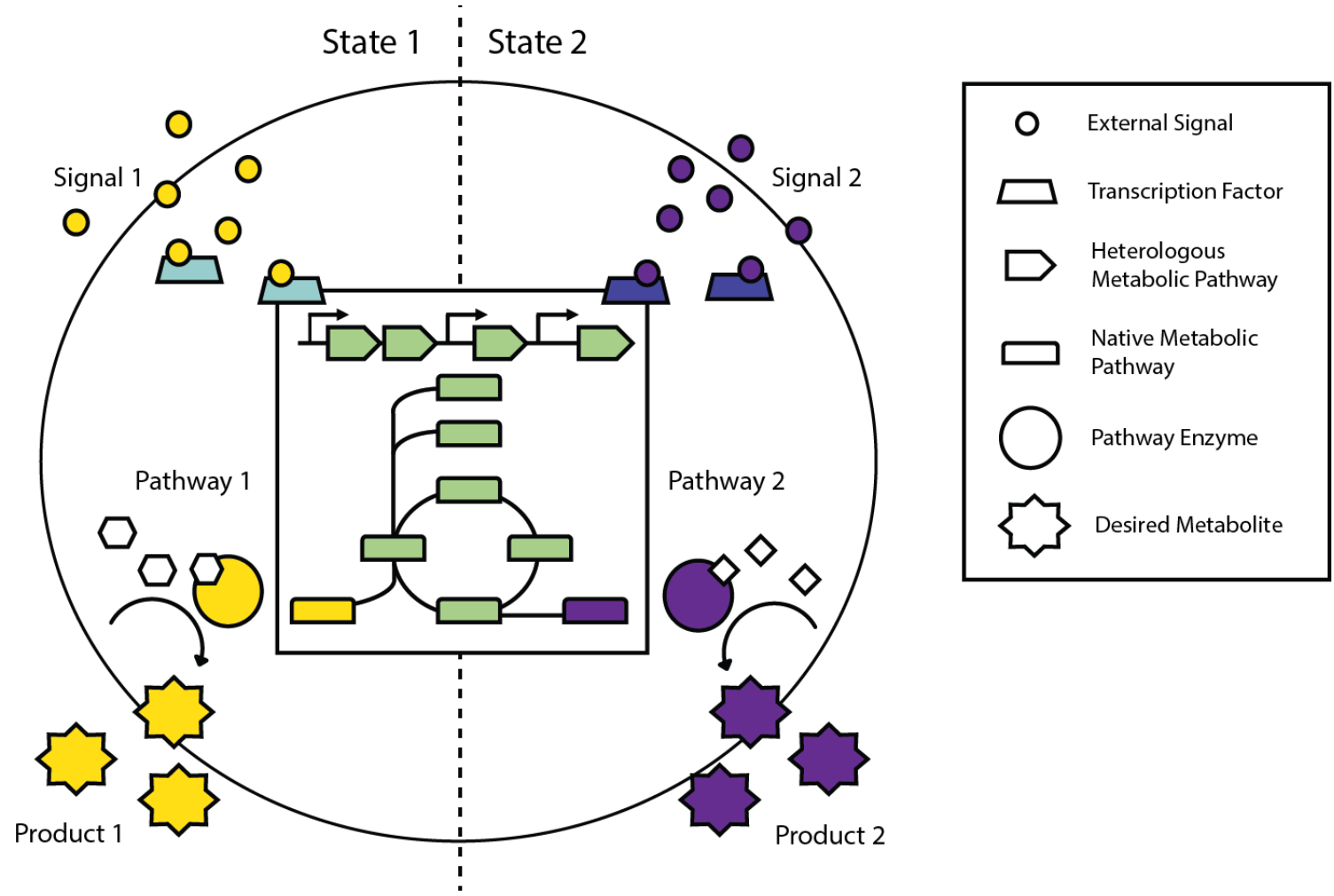

Figure 1: Schematic of precision metabolic engineering

For precision metabolic engineering, cells must be capable of sensing different signals and, through robust cell regulation of native and heterologous metabolic pathways, ensure that only the desired products are made. In this example, external signals (small circles) enter the cell and bind transcription factors (trapezoids). The activated transcription factors control cell metabolism (central schematic in box) by affecting transcription from either native or heterologous metabolic pathways. Pathway enzymes (large circles) produce the desired metabolites (starburst shapes), and control mechanisms ensure high product selectivity over metabolites of competing pathways.

\section{Sensory mechanisms to determine the state of the cell}

A key characteristic of precision metabolic engineering is the production of a signalspecific metabolic response; to produce a specific response, a cell must first sense that signal. Whole cell biosensors have been designed to sense environmental signals and specific chemicals (Zhang and Keasling, 2011), and recent reviews have highlighted how dynamic metabolic engineering systems have been designed to similarly sense small molecules and metabolites, 
144 responding to inducers added at specific points in the production process or to a specific 145 concentration of a naturally occurring molecule that changes over time (Brockman and Prather, 146 2015a; Venayak et al., 2015). Though these sensors are explicitly designed to be activated at a 147 time point within the process, the same design approaches can be used to make sensors that do not 148 necessarily effect changes in time.

\subsection{Small molecule inducers}

Exogenous inducers have been used in several dynamic systems to initiate a switch from a 152 growth phase to a production phase (Brockman and Prather, 2015b; Davis et al., 2011; Soma et 153 al., 2014; Torella et al., 2013). Small molecules like allolactose analogs (IPTG) and

154 anhydrotetracycline have well-characterized interactions with promoter-repressor pairs and are 155 widely used. Genetic circuits can be designed such that genes critical to growth but detrimental to 156 production of a commercial target are expressed during the growth phase and repressed upon 157 addition of an inducer. Optimal inducer concentration and time of induction can be determined to 158 maximize titer and productivity.

159 The use of exogenous inducers could be useful in precision applications in which the user 160 knows what the system should be producing. For example, microbes capable of producing a range 161 of pharmaceuticals based on demand could produce specific pharmaceuticals in response to 162 specific exogenous inducers. Exogenous inducers have less utility in biosensor applications, since 163 the system must respond to a specific environmental signal. However, they could be used to 164 activate or repress a sensory system: if sensory systems need to be off during the mass production 165 of biosensor cells for later use in assays, then the sensory and response systems could be repressed 166 through small molecule-responsive promoters and repressors, to be turned on only when desired.

\subsection{Autonomous sensory systems}

169 Cells have many natural mechanisms to sense and respond to their surroundings, and 170 dynamic metabolic engineering has begun to harness this cellular machinery for use in controlled 171 autonomous regulation (Carter et al., 2012). One commonly used mechanism is quorum sensing,

172 the process through which cells secrete and sense autoinducers to sense their population density.

$173 E$. coli have been engineered to sense and respond to quorum sensing molecules of their own and 174 of different species (Kobayashi et al., 2004; Tsao et al., 2010) by expressing genes under quorum- 
175 responsive promoters. Similarly, the nitrogen regulatory system in E. coli was rewired to initiate 176 the production of heterologous proteins upon sensing of acetyl-phosphate (Farmer and Liao, 2000)

177 and acetate (Bulter et al., 2004), signals of excess metabolic flux that indicated cells were 178 approaching the late exponential growth phase.

Natural methods to sense population density could replace small molecule induction in industrial processes where redirection of metabolic flux is required upon reaching a certain cell density. In the general case of precision metabolic engineering, in which production does not necessarily change over time, quorum sensing may have limited utility. However, quorum sensing could be useful in a subset of problems in which cells must modulate their response based on the number of cells present. For example, as discussed earlier, one challenge to using microbes to treat cancer is the inherent toxicity of bacteria and infections that can occur if bacterial growth cannot be controlled (Forbes, 2010). Quorum sensing could be used to trigger bacterial self-destruction upon reaching a threshold cell density, in this case enforcing precision on the dose of therapeutic cells in addition to the synthesis of their pharmaceutical products.

The same methods used to engineer specific quorum responses can be used to harness any natural transcriptional regulatory mechanism of a cell. Cells have transcriptional regulatory mechanisms to sense and respond to relative concentrations of different nutrients, ions, and sugars present in the cell (Chantranupong et al., 2015), and these sensory systems could be engineered to differentially regulate metabolism in response to different levels of analytes in the system.

\subsection{Protein engineering to alter receptor-ligand affinity and selectivity}

In the presence of known regulator-promoter interactions, protein engineering can be used to affect a sensor's dynamic range by modulating regulator-substrate affinity (Cobb et al., 2013).

198 Transcription factors involved in quorum sensing have been the target of extensive protein engineering efforts to accomplish such goals (Collins et al., 2005; Hawkins et al., 2007; Kambam et al., 2008, 2009). For example, by screening a large library of LuxR mutants, a library of

201 transcription factors with up to a 100 fold increase in sensitivity to specific molecules was developed (Collins et al., 2005). Similar approaches have been successful in changing the dynamic

203 range of other ligand-regulated transcription factors (Michener et al., 2012). Protein engineering 204 can also change substrate specificity, allowing the construction of entirely new 'sensors' from 
screening of a mutant library if the protein structure is well-characterized, rational, directed

207 mutagenesis of amino acids can effectively change receptor-ligand affinity and selectivity (Khan

208 et al., 2002).

Protein engineering thus has significant potential to enhance responses to specific signals so that the dynamic sensing range corresponds with relevant concentrations of analyte (see also

211 Section 4). Combined with the broad range of natural sensory mechanisms available across 212 different organisms, this could allow a metabolic system to respond to an increasing number of 213 external signals, whether exogenous or endogenous to the system.

\section{Tight control of signal transduction through metabolic pathways}

224

225

Another hallmark of precision metabolic engineering is tight control of metabolic state based on the signals that are sensed. A recently growing approach in metabolic engineering is the use of metabolite valves, which redirect metabolic flux from central carbon metabolic pathways to competing production pathways (Solomon et al., 2012; Soma et al., 2014). Though these valves shift the bulk of metabolic flux, some baseline expression through competing pathways can be permitted, which is often problematic in precision applications. A similar approach can be applied in a precision metabolic engineering context, where instead of just diverting flux, competing valves must be completely closed upon determination of state (Figure 2).

a) Dynamic Metabolic Engineering

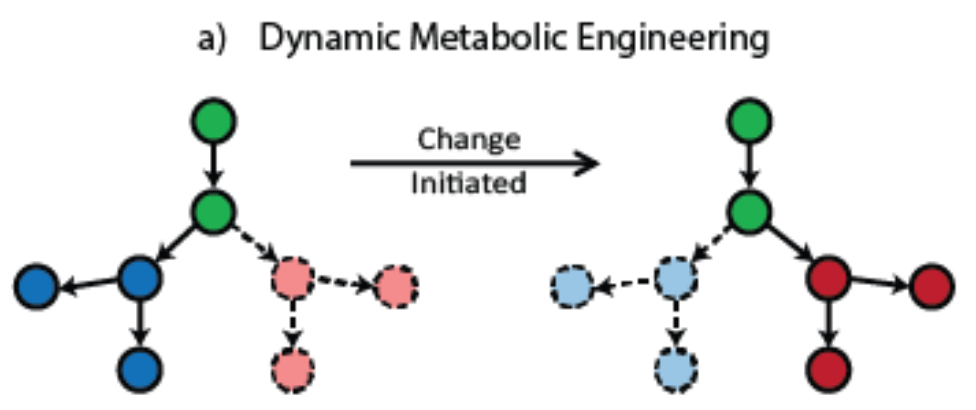

Figure 2: Direction of flux in dynamic and precision metabolic engineering

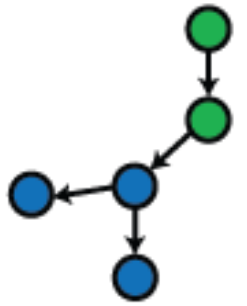

State1

b) Precision Metabolic Engineering

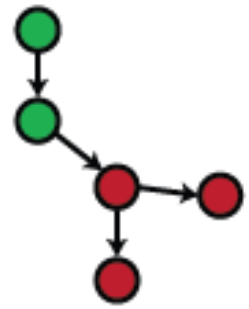

State 2 A) Dynamic control redirects flux based on a change in the system. The pathway flux distribution changes, but flux may still be permitted through competing pathways. Initially, the majority of the metabolic flux is through the left branch of the pathway, with some flux permitted through the right branch. Upon initiation of a change, the flux shifts so that the majority is through the right branch. Circles represent metabolites in the pathway, with 
solid circles indicating significant production of a metabolite and dotted circles indicating partial repression of its production.

B) Rather than emphasizing the ability to dynamically switch between metabolic states, precision metabolic engineering emphasizes the completeness of the switch in metabolic state. Competing pathways are completely repressed so that only the desired metabolic pathway is active. mild growth during the production phase (Soma et al., 2014), which could in some situations be advantageous. However, in situations where complete control of metabolic flux is required, even minimal leakage can cause problems, and layers of regulation will likely be necessary to ensure that flux is completely redirected from competing pathways. Transcriptional, translational, and post-translational methods can be used, often in combination, to completely control flux through 245 pathways.

\subsection{Promoter engineering to tighten transcriptional regulation}

E. coli has numerous known transcriptional regulatory systems in which the interaction of a transcription factor with an external signal can change the rate of transcription of different genes. However, the regulators may not be sufficiently strong to tightly control (induce or repress transcription of) engineered pathways. Promoter engineering can be used to enable transcriptional regulators to more strongly affect transcription. For example, promoters were designed to increase the regulatory capabilities of FadR (Zhang et al., 2012) and FapR (Xu et al., 2014a), transcription factors that respond to the fatty acid precursors acyl-CoA and malonyl-CoA, respectively. Zhang et al. integrated the FadR operator into two locations in the phage lambda and phage T7 promoters and demonstrated a 60 fold increase in the system's response to acyl-CoA (Zhang et al., 2012). Xu et al. had similar success integrating the FapR operator into a T7 promoter to increase its response to malonyl-CoA (Xu et al., 2014a).

In designing promoters to be more strongly repressible, predicting regulator-polymerase interactions is difficult. When trying to make a second promoter that would also be active in the presence of malonyl-CoA, $\mathrm{Xu}$ et al. (Xu et al., 2014b) designed a promoter that was in fact repressed upon malonyl-CoA binding to the transcription factor. This ultimately proved 
263 beneficial - the two promoters with opposite responses to malonyl-CoA could differentially 264 regulate genes in the presence of malonyl-CoA-but demonstrates the unpredictability of the 265 effects of different regulators. Adding operators to different promoters can increase transcriptional 266 regulation, but it cannot change the affinity with which the regulator binds DNA; without protein 267 engineering, this method may not be effectively extrapolated to transcriptional regulators that interact weakly with DNA.

To help reduce leaky expression, both groups designed hybrid promoters that were repressed by the native transcription factor and LacI. This added an extra layer of control to the system: expression from the promoter would only occur if both the fatty acid precursor and IPTG

272 were present in the system. This created a transcriptional AND gate, which is of importance in 273 synthetic biology and biological computing and could have an increasingly important role in metabolic engineering as systems are designed to specifically respond to different combinations of signals. For example, in drug delivery applications, requiring two signaling molecules to be present instead of one could decrease nonselective delivery of a toxic drug.

While promoter engineering has significant potential to help redirect flux, limitations imposed by desired regulators could render this strategy alone insufficient. In these cases,

\subsection{CRISPR Transcriptional Regulation}

Recently, the CRISPR/Cas system was demonstrated to be a powerful tool for genome engineering (Cong et al., 2013; Mali et al., 2013). With some modifications, CRISPR/Cas systems can also be used for metabolic regulation. CRISPR interference (CRISPRi) has been used as a gene knock down tool: catalytically inactive Cas9 (dCas9) brings a guide RNA to the chromosome and unwinds the DNA through helicase activity. Upon reaching the gene of interest, the guide RNA binds to its complementary DNA strand, and dCas9 sterically interferes to prevent transcription of the gene (Qi et al., 2013). Transcriptional effectors can be fused to dCas9 to further repress or activate transcription from adjacent genes (Gilbert et al., 2013). Alternatively, guide RNA can also be extended with modular RNA domains to recruit transcriptional regulators via

292 Generally, inducing the expression of dCas9 can turn on all parts of CRISPR regulation and tightly 293 control the enzymatic activity of complex systems. Orthogonal dCas9 proteins that recognize 
294 different RNA sequences (Esvelt et al., 2013) could be induced under different conditions to 295 differentially regulate genes (Zalatan et al., 2015).

296 Regulation with CRISPR/Cas9 could be useful in precisely directing metabolic flux 297 because of its high selectivity for its target gene, use of orthogonal Cas9 proteins as master 298 regulators, and limited interference with natural cellular pathways. CRISPR/Cas9 regulation 299 completely repressed competing pathways in the branched violacein pathway, demonstrating its 300 ability to tightly control which products are produced (Zalatan et al., 2015). Since CRISPR/Cas9 301 enables robust genomic regulation, it can also provide an alternative to strictly plasmid-based heterologous expression and regulation, which has the disadvantages of metabolic burden and 303 unstable propagation characteristics (Anthony et al., 2009; Bentley et al., 1990; Diaz Ricci and 304 Hernández, 2000).

\subsection{Synthetic biology methods to control transcription}

The field of synthetic biology has many examples of novel methods to turn cellular responses on and off, many of which have been used in metabolic engineering. The development of more tightly controlled synthetic biology devices could enable more precise control of metabolism. The genetic toggle switch (Gardner et al., 2000) has been used in dynamic metabolic engineering applications to generate "on" and "off" states through the use of well-characterized promoter-regulator interactions (Soma et al., 2014), and more elaborate switches can be made by layering logic gates (Moon et al., 2012). In a basic synthetic biology circuit, toggle switches can enable nearly complete shifts between states, but when controlling more complex circuits, toggle switches are not always effective at eliminating leaky expression and completely repressing alternative pathways (Soma et al., 2014). Applying these techniques to existing sensors and regulators could help enable more complete switch-like behavior, but their limitations would need to be addressed for direct application in precision metabolic engineering.

Riboregulators have shown the potential to regulate metabolic flux more precisely than

321 to a ribosomal binding site in the untranslated region. Upon transcription, this sequence binds to 322 the RBS and forms a hairpin to prevent RNA translation. A short noncoding RNA sequence, 323 expressed from a different promoter, interacts with the interfering RNA to expose the RBS and 324 allow protein translation (Isaacs et al., 2004). They demonstrated some of this system's major 
advantages: leakage minimization, tunable gene expression, fast response time, and independent

326 regulation of multiple genes (Callura et al., 2010), all of which make this a very attractive 327 regulatory mechanism to precisely control metabolism. A layer of complexity was added to 328 riboregulator regulation through a switchboard that can detect and produce unique responses to a 329 theoretically infinite number of signals; they demonstrated its ability to control metabolic flux 330 through a branched pathway and prevent unwanted enzyme activity (Callura et al., 2012). 331 Practically, this approach is limited by the small number of characterized natural receptor332 promoter pairs, but it still provides an excellent framework for tight, synthetic biology-based 333 control of transcription.

\subsection{Post-transcriptional regulation methods}

While transcriptional control is useful, control of translation is an equally important way to control cellular state, since it is ultimately enzyme activity that controls cellular metabolism. Dynamic metabolic engineering efforts have previously used interfering RNA (Williams et al., 2015) to prevent translation of specific genes. Precision metabolic engineering must go beyond preventing translation of unwanted proteins, to controlling exactly how much individual proteins are translated. Tools used for flux balancing and in synthetic biology can be used to accomplish 342 this.

To effectively control protein concentration, several levels of post-transcriptional control will likely be necessary. Altering the ribosomal binding site of individual proteins can change relative protein translation efficiency (Salis et al., 2009), changing gene order within an operon can change relative translation rates of different proteins (Lee et al., 2013), and changing the 3' untranslated region of messenger RNA can affect RNA stability (Lu et al., 2011; Zhao et al., 2013).

348 Varying tunable intergenic regions between genes can alter RNA stability and ribosomal binding 349 site availability independent of RBS strength (Pfleger et al., 2006). Protein expression can be 350 altered after translation by adding different degradation tags to change the rate at which specific 351 proteins are degraded (McGinness et al., 2006). Taken together, these constitute a powerful suite 352 of tools for post-transcriptional control in precision metabolic engineering.

\subsection{Dynamic protein degradation}

While degradation tags are usually used to permanently target proteins for degradation, 
inducible expression of the degradation machinery can be used to selectively enhance protein

357 degradation only in certain system (signal) states. This is a useful tool in dynamic metabolic 358 engineering, as it can increase the rate at which enzymes critical for growth are degraded so that 359 metabolic flux can be directed to production pathways. Knockout of $s s p B$ (the gene responsible 360 for tethering tagged proteins to E. coli's ClpXP degradation system) can prevent protein 361 degradation, so inducible complementation of $\operatorname{ssp} B$ expression at a specific time can allow for 362 temporally controlled degradation of tagged proteins (Brockman and Prather, 2015b; Davis et al., 363 2011; Torella et al., 2013). Since knockout and induction of $\operatorname{sspB}$ could have deleterious effects 364 on cell metabolism, as it disrupts natural protein degradation functions, inducible heterologous 365 protein degradation systems have also been explored. An ssrA tag and protease from M. florum 366 have been successfully used to orthogonally control protein degradation in E. coli (Cameron and 367 Collins, 2014): E. coli's ClpXP system does not recognize the heterologous tag, and the 368 heterologous protease does not disrupt E. coli's native ClpXP-ssrA degradation system (Cameron and Collins, 2014).

As noted above, inducible protein degradation has been used in dynamic metabolic engineering as a stand-alone method to decrease the concentration of pivotal enzymes (Brockman and Prather, 2015b; Davis et al., 2011; Torella et al., 2013). However, tag-based degradation

373 (whether induced or constitutive) is never complete (there is always some recently translated 374 protein having some potentially undesirable enzymatic activity), and expressing an unwanted 375 protein just to quickly degrade it can place a significant metabolic burden on the cell. Thus, in 376 precision metabolic engineering, protein degradation (through either native or heterologous systems) might instead be used as a secondary level of control. Transcription of competing pathways could be repressed, and if leaky expression of genes is problematic, the unwanted proteins could be degraded to reduce unwanted enzyme expression.

\section{Engineering response hypersensitivity to specified signal concentrations}

For a system to properly respond to a specific input, it must be calibrated so that it responds to the correct concentration of signal in a sharp, switch-like way. The system should ideally respond with either one metabolic mode or another, rather than with intermediate or gradient-like responses that are often present in natural biological and metabolic systems. Biosensor and drug delivery devices require sharp switch-like behavior to prevent ambiguous sensor readings and 
unwanted delivery of toxic drugs. Many of the transcriptional and post-transcriptional methods

388 described in Section 3 can be used to tune the set point of the response and to facilitate sharper responses; those will not be rehashed in this section. Instead, we will focus on additional approaches that are useful for tuning the sharpness and set point of the response, including precursor availability and gene dosage.

\subsection{Tuning precursor availability}

Since the rate at which an enzyme converts metabolites often scales with the concentration of metabolite present, metabolic flux can be adjusted by modulating the concentration of precursors. This approach could then increase the sharpness or signaling set point of a response.

Controlling precursor concentration is not a new idea in metabolic engineering. Increasing precursor availability is an effective approach used widely across the field of metabolic engineering to increase titer for pathways that are not already saturated with flux. In addition, it has been used in balancing flux to prevent accumulation of toxic intermediates. Tunable intergenic regions (Pfleger et al., 2006), protein scaffolds (Dueber et al., 2009), addition of pathways (Pitera et al., 2007), and pathway balancing with metabolite responsive promoters (Dahl et al., 2013) have all been used to minimize the build-up of toxic metabolites.

A prototypical example demonstrating this concept for precision metabolic engineering is our recently described pigment-based biosensor (Watstein et al., 2015). In this work, two pigments from the same pathway (lycopene and $\beta$-carotene) were used as indicators, with conversion between them by the gene $\operatorname{crt} Y$ controlled by a signal-responsive transcription factor. However, flux to the entry point of the pathway was low, which meant that slightly leaky expression of $\operatorname{crtY}$

410 lycopene to $\beta$-carotene. By supplementing with the mevalonate pathway, total flux to the pathway

411 was increased, rendering the low $\operatorname{crt} Y$ expression negligible, and allowing the lycopene-producing 412 state to be accessible. Moreover, for circuit configurations already with switch-like behavior, 413 manipulating precursor availability tuned the set point for the switch relative to sensor input.

\subsection{Regulating gene dosage}

In addition to modulation of precursor levels, fluxes can also be regulated by changing 
chromosomally integrated. Modular metabolic engineering has successfully used this method to

419 eliminate flux imbalances by grouping pathway enzymes with a lower turnover rate on a higher 420 copy plasmid to make the turnover rates of all enzymes more equal (Yadav et al., 2012). By varying 421 plasmid copy number of different modules and adjusting promoter strengths, Ajikumar et al. 422 achieved a 15,000 fold greater titer of taxane than through previous production methods (Ajikumar 423 et al., 2010). This approach can also be useful for plasmid-based systems with regulatory elements 424 (cis or trans) that are not orthogonal to the host: cross-talk interactions that are detrimental to the 425 host or interfere with the metabolic program being added can be titrated out by changing the relative contributions of native versus plasmid-based regulatory elements.

\subsection{Rational optimization methods}

Tuning systems to sharply respond to a specific concentration of signal will likely require multiple levels of regulation, and determining the optimum combination of parts can be very difficult. It is often possible to create combinatorial libraries of regulated constructs, but assessing the performance of such libraries typically entails the use of high-throughput screens, which are not necessarily available for arbitrary phenotypes. Lee et al. used limited testing of a library combined with regression algorithms to predict the optimal combination of regulatory elements for a given phenotype, eliminating the need for a high-throughput screen (Lee et al., 2013) (though they still had to create the library).

As an alternative, multivariate modular metabolic engineering (MMME) has been used to maximize yield of a desired product by breaking a complicated system into modules and using transcriptional, translational, and post-translational modification methods to determine the optimum expression levels of each module. By treating a complicated system as a series of simpler, independent subsystems, a multivariate statistical analysis can be used to determine the optimal configuration so that extensive combinations of parts do not have to be made and tested (Yadav et al., 2012).

This multivariate approach could also be used to enhance precise control of metabolic systems. Rational design of individual modules could remove the need for high-throughput screening and enable more efficient combination of optimized modules. Variables would not be used to eliminate bottlenecks and balance flux, but rather to eliminate unwanted protein expression, increase sharpness of the response, and tune the set point (Figure 3). 
a)
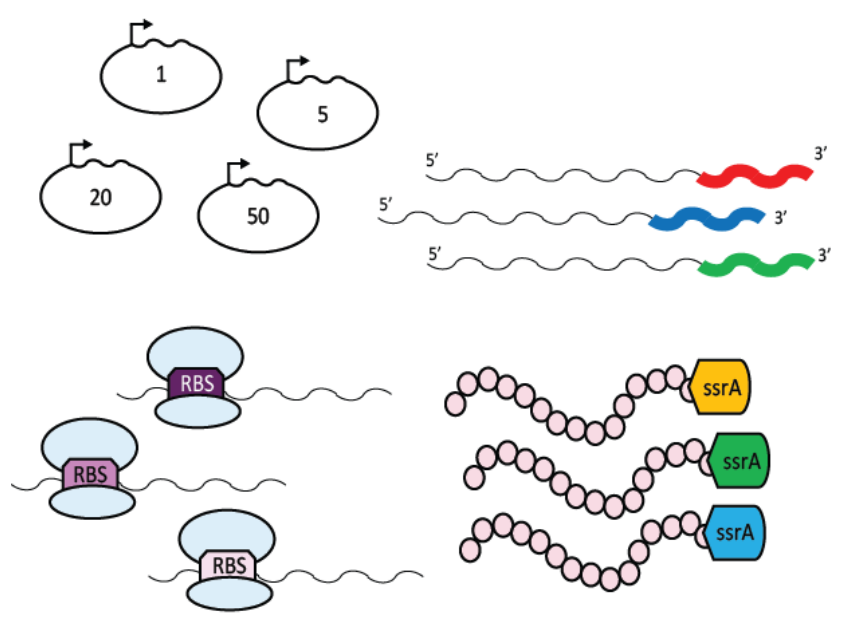

b)

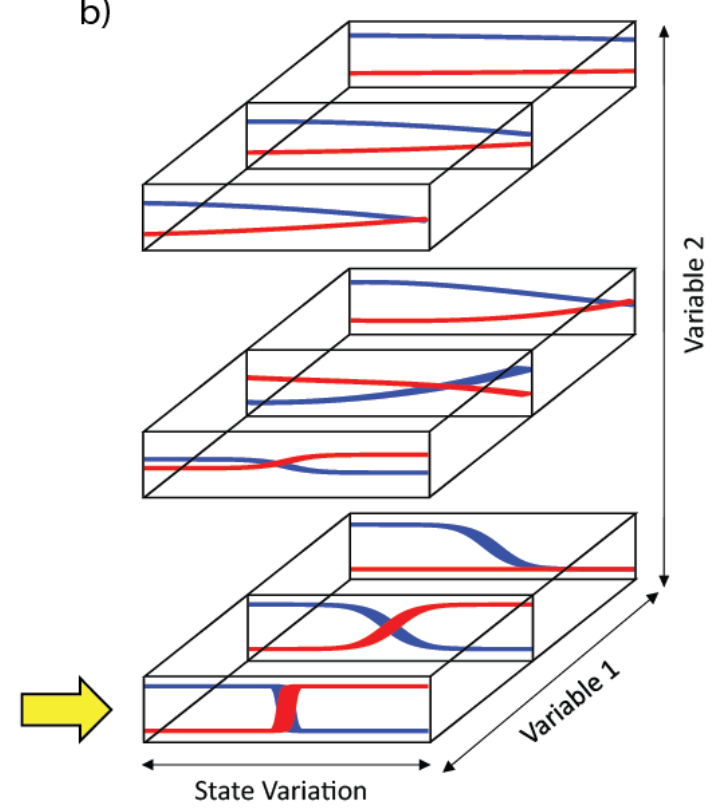

State Variation

Figure 3: Multivariate optimization for precision metabolic engineering. Adapted from Yadav et al. (Yadav et al., 2012)

A) Variables in precision metabolic engineering could include plasmid copy number, mRNA stabilizing regions, RBS strength, and protein degradation tags of the modules to be tuned

B) A two-variable optimization scheme. Variables 1 and 2 are changed to optimize the statebased selectivity and sharpness of the system response. The optimum combination, indicated by the yellow arrow, sharply responds to a change in state and has a switch point in an appropriate region.

promoter strength for different parts, variation of promoters is not always an option. When promoter-regulator interactions are fixed, it may be impossible to alter promoter strength without losing regulator effectiveness (Xu et al., 2013). In these cases, post-transcriptional regulation is necessary, and variables to be optimized can potentially include copy number, ribosomal binding site sequence, protein degradation tag, and tunable intergenic regions.

More tools for MMME are continuously being developed. Libraries of characterized parts (Alper et al., 2005b; Pfleger et al., 2006; Zaslaver et al., 2006) have decreased the number of combinations required to be tested for effective MMME, and predictive algorithms (Salis, 2011; 
Salis et al., 2009) can further decrease the range of variation required to achieve optimal 470 expression. A recent review (Biggs et al., 2014) discusses the need to expand and further 471 characterize the tools available for tuning in MMME. As the MMME toolset expands, precision 472 metabolic engineering will be able to be more easily applied to other systems.

\section{Conclusions}

By using existing techniques in new ways, metabolic engineering can expand its applications to produce flexible systems capable of sensing their environment and responding to different stimuli with distinct metabolic programs. In applications that require extreme product selectivity, tight control mechanisms must be in place to ensure that only the desired product is made. This is similar to (but a departure from) dynamic metabolic engineering, which focuses on temporarily diverting flux from, but not necessarily shutting down, competing pathways. While exogenous small molecule induction that is useful in dynamic applications has limited utility in precision metabolic engineering, autonomous sensory mechanisms and methods used to enhance their dynamic range and selectivity can be used to develop sensors for precision metabolic engineering applications. Transcriptional and post-transcriptional methods of controlling metabolic flux can be used in combination to ensure complete pathway selectivity. Of course, the goal of 100\% complete elimination of anything in a biological system may be an impractical ideal; in the context of precision metabolic engineering, the point is that the $10 \%$ or $1 \%$ leakiness that is easy to achieve and is permissible in many applications can be quite problematic, requiring much tighter control than is typically pursued in metabolic engineering or synthetic biology applications.

Precision metabolic engineering will almost always require extensive tuning of metabolic responses. Standard methods of promoter engineering to change transcription rate are not viable options when the promoter to be used is dictated by sensor-regulator interactions. Protein engineering can be used to increase regulator affinity and dynamic range, but more typically posttranscriptional regulation would be used to control translation rate and protein degradation. Multivariate modular metabolic engineering provides a potentially valuable framework for the rational variation of multiple components to efficiently optimize systems.

There are a number of ways that future technological developments would address the current challenges of precision metabolic engineering. One of the main limits on the application of precision metabolic engineering is the limited number of well-characterized sensory-regulator 
500 systems available, such that developing new sensory systems would be extremely valuable. 501 Aptamers, which are DNA or RNA sequences that can specifically bind small molecules and can 502 be coupled with other elements to selectively induce enzymatic or regulatory activity, show 503 significant potential in helping combat this challenge. In particular, aptamers used in combination 504 with riboregulators could create tightly controlled systems that could specifically respond to 505 different molecular signals. To date, the effort and difficulty associated with creating aptamers that 506 bind specifically to small molecules of interest has limited their wide-spread use. However, recent 507 improvements on high-throughput technologies to develop sensitive and specific aptamers (Cho 508 et al., 2013; Wang et al., 2014) will likely hasten creation of novel aptamers, potentially broadening 509 the scope of who can reasonably develop new aptamers and increasing the use of aptamer-based 510 regulation. Other significant challenges to be addressed for precision metabolic engineering 511 include reducing the experimental burden of tuning the system to respond in a switch-like way at 512 a specified signal threshold. Computational models have been developed in dynamic metabolic 513 engineering to predict the ways that changing time of induction and other controllable parameters 514 would affect productivity (Anesiadis et al., 2008, 2013; Gadkar et al., 2005), and similar models 515 could be developed to predict the ways that the switching point and switch-like behavior of a 516 system could be optimized.

517 We expect the number of precision metabolic engineering applications to increase as 518 scientists develop more and more complex tasks for microbes to complete. While existing 519 examples of precision metabolic engineering - including using microbes as micronutrient 520 biosensors, portable pharmaceutical platforms, and drug delivery vehicles - are exciting and have 521 great potential, they are likely just the beginning of ways that metabolic engineers will look to 522 precisely control cell state and function.

\section{Acknowledgments}

525 The authors acknowledge the Bill \& Melinda Gates Foundation for funding support 526 (OPP1046289), as well as the National Science Foundation (1254382). MPM was supported by an 527 NIH training grant (T32-EB0064343). 


\section{References}

530 Ajikumar, P.K., Xiao, W.-H., Tyo, K.E.J., Wang, Y., Simeon, F., Leonard, E., Mucha, O., Phon, T.H., Pfeifer, B., and Stephanopoulos, G. (2010). Isoprenoid Pathway Optimization for Taxol Precursor Overproduction in Escherichia coli. Science 330, 70-74.

Alonso-Gutierrez, J., Chan, R., Batth, T.S., Adams, P.D., Keasling, J.D., Petzold, C.J., and Lee, T.S. (2013). Metabolic engineering of Escherichia coli for limonene and perillyl alcohol production. Metab. Eng. 19, 33-41.

Alper, H., Miyaoku, K., and Stephanopoulos, G. (2005a). Construction of lycopeneoverproducing E. coli strains by combining systematic and combinatorial gene knockout targets. Nat. Biotechnol. 23, 612-616.

Alper, H., Fischer, C., Nevoigt, E., and Stephanopoulos, G. (2005b). Tuning genetic control through promoter engineering. Proc. Natl. Acad. Sci. U. S. A. 102, 12678-12683.

Anesiadis, N., Cluett, W.R., and Mahadevan, R. (2008). Dynamic metabolic engineering for increasing bioprocess productivity. Metab. Eng. 10, 255-266.

Anesiadis, N., Kobayashi, H., Cluett, W.R., and Mahadevan, R. (2013). Analysis and Design of a Genetic Circuit for Dynamic Metabolic Engineering. ACS Synth. Biol. 2, 442-452.

Atsumi, S., Hanai, T., and Liao, J.C. (2008b). Non-fermentative pathways for synthesis of branched-chain higher alcohols as biofuels. Nature 451, 86-89.

Anthony, J.R., Anthony, L.C., Nowroozi, F., Kwon, G., Newman, J.D., and Keasling, J.D. (2009). Optimization of the mevalonate-based isoprenoid biosynthetic pathway in Escherichia coli for production of the anti-malarial drug precursor amorpha-4,11-diene. Metab. Eng. 11, 13-19.

Atsumi, S., Cann, A.F., Connor, M.R., Shen, C.R., Smith, K.M., Brynildsen, M.P., Chou, K.J.Y., Hanai, T., and Liao, J.C. (2008a). Metabolic engineering of Escherichia coli for 1-butanol production. Metab. Eng. 10, 305-311.

Biggs, B.W., De Paepe, B., Santos, C.N.S., De Mey, M., and Kumaran Ajikumar, P. (2014). Multivariate modular metabolic engineering for pathway and strain optimization. Curr. Opin. Biotechnol. 29, 156-162. 
Brockman, I.M., and Prather, K.L.J. (2015a). Dynamic metabolic engineering: New strategies for developing responsive cell factories. Biotechnol. J. n/a - n/a.

Brockman, I.M., and Prather, K.L.J. (2015b). Dynamic knockdown of E. coli central metabolism for redirecting fluxes of primary metabolites. Metab. Eng. 28, 104-113.

Brown, J.M., and Wilson, W.R. (2004). Exploiting tumour hypoxia in cancer treatment. Nat. Rev. Cancer 4, 437-447.

Bulter, T., Lee, S.-G., Wong, W.W., Fung, E., Connor, M.R., and Liao, J.C. (2004). Design of artificial cell-cell communication using gene and metabolic networks. Proc. Natl. Acad. Sci. U. S. A. 101, 2299-2304.

Callura, J.M., Dwyer, D.J., Isaacs, F.J., Cantor, C.R., and Collins, J.J. (2010). Tracking, tuning, and terminating microbial physiology using synthetic riboregulators. Proc. Natl. Acad. Sci. 107, 15898-15903.

Callura, J.M., Cantor, C.R., and Collins, J.J. (2012). Genetic switchboard for synthetic biology applications. Proc. Natl. Acad. Sci. 109, 5850-5855.

Cameron, D.E., and Collins, J.J. (2014). Tunable protein degradation in bacteria. Nat. Biotechnol. 32, 1276-1281.

Carter, K.K., Valdes, J.J., and Bentley, W.E. (2012). Pathway engineering via quorum sensing and sRNA riboregulators-interconnected networks and controllers. Metab. Eng. 14, 281288.

Chantranupong, L., Wolfson, R.L., and Sabatini, D.M. (2015). Nutrient-Sensing Mechanisms across Evolution. Cell 161, 67-83.

Chemler, J.A., Fowler, Z.L., McHugh, K.P., and Koffas, M.A.G. (2010). Improving NADPH availability for natural product biosynthesis in Escherichia coli by metabolic engineering. Metab. Eng. 12, 96-104.

Cheol Gil, G., Mitchell, R.J., Tai Chang, S., and Bock Gu, M. (2000). A biosensor for the detection of gas toxicity using a recombinant bioluminescent bacterium. Biosens. Bioelectron. 15, 23-30.

Cho, M., Soo Oh, S., Nie, J., Stewart, R., Eisenstein, M., Chambers, J., Marth, J.D., Walker, F., Thomson, J.A., and Soh, H.T. (2013). Quantitative selection and parallel characterization of aptamers. Proc. Natl. Acad. Sci. U. S. A. 110, 18460-18465. 
Choi, E.J., and Ling, G.S.F. (2014). Battlefield Medicine: Paradigm Shift for Pharmaceuticals Manufacturing. PDA J. Pharm. Sci. Technol. 68, 312-312.

Cobb, R.E., Sun, N., and Zhao, H. (2013). Directed evolution as a powerful synthetic biology tool. Methods San Diego Calif 60, 81-90.

Collins, C.H., Arnold, F.H., and Leadbetter, J.R. (2005). Directed evolution of Vibrio fischeri LuxR for increased sensitivity to a broad spectrum of acyl-homoserine lactones. Mol. Microbiol. 55, 712-723.

Cong, L., Ran, F.A., Cox, D., Lin, S., Barretto, R., Habib, N., Hsu, P.D., Wu, X., Jiang, W., Marraffini, L.A., et al. (2013). Multiplex Genome Engineering Using CRISPR/Cas Systems. Science 339, 819-823.

Dahl, R.H., Zhang, F., Alonso-Gutierrez, J., Baidoo, E., Batth, T.S., Redding-Johanson, A.M., Petzold, C.J., Mukhopadhyay, A., Lee, T.S., Adams, P.D., et al. (2013). Engineering dynamic pathway regulation using stress-response promoters. Nat. Biotechnol. 31, 10391046.

Davis, J.H., Baker, T.A., and Sauer, R.T. (2011). Small-molecule control of protein degradation using split adaptors. ACS Chem. Biol. 6, 1205-1213.

Diaz Ricci, J.C., and Hernández, M.E. (2000). Plasmid effects on Escherichia coli metabolism. Crit. Rev. Biotechnol. 20, 79-108.

Dietrich, J.A., McKee, A.E., and Keasling, J.D. (2010). High-Throughput Metabolic Engineering: Advances in Small-Molecule Screening and Selection. Annu. Rev. Biochem. 79, 563-590.

Dueber, J.E., Wu, G.C., Malmirchegini, G.R., Moon, T.S., Petzold, C.J., Ullal, A.V., Prather, K.L.J., and Keasling, J.D. (2009). Synthetic protein scaffolds provide modular control over metabolic flux. Nat. Biotechnol. 27, 753-U107.

Esvelt, K.M., Mali, P., Braff, J.L., Moosburner, M., Yaung, S.J., and Church, G.M. (2013). Orthogonal Cas9 proteins for RNA-guided gene regulation and editing. Nat. Methods 10, 1116-1121.

Fang, M.-Y., Zhang, C., Yang, S., Cui, J.-Y., Jiang, P.-X., Lou, K., Wachi, M., and Xing, X.-H. (2015). High crude violacein production from glucose by Escherichia coli engineered with interactive control of tryptophan pathway and violacein biosynthetic pathway. Microb. Cell Factories 14, 8. 
621 Farmer, W.R., and Liao, J.C. (2000). Improving lycopene production in Escherichia coli by engineering metabolic control. Nat. Biotechnol. 18, 533-537.

Forbes, N.S. (2010). Engineering the perfect (bacterial) cancer therapy. Nat. Rev. Cancer 10, 785-794.

Gadkar, K.G., Doyle Iii, F.J., Edwards, J.S., and Mahadevan, R. (2005). Estimating optimal profiles of genetic alterations using constraint-based models. Biotechnol. Bioeng. 89, 243-251.

Gardner, T.S., Cantor, C.R., and Collins, J.J. (2000). Construction of a genetic toggle switch in Escherichia coli. Nature 403, 339-342.

Gilbert, L.A., Larson, M.H., Morsut, L., Liu, Z., Brar, G.A., Torres, S.E., Stern-Ginossar, N., Brandman, O., Whitehead, E.H., Doudna, J.A., et al. (2013). CRISPR-mediated modular RNA-guided regulation of transcription in eukaryotes. Cell 154, 442-451.

Hawkins, A.C., Arnold, F.H., Stuermer, R., Hauer, B., and Leadbetter, J.R. (2007). Directed Evolution of Vibrio fischeri LuxR for Improved Response to Butanoyl-Homoserine Lactone. Appl. Environ. Microbiol. 73, 5775-5781.

Isaacs, F.J., Dwyer, D.J., Ding, C., Pervouchine, D.D., Cantor, C.R., and Collins, J.J. (2004). Engineered riboregulators enable post-transcriptional control of gene expression. Nat. Biotechnol. 22, 841-847.

Jin, Y.-S., Alper, H., Yang, Y.-T., and Stephanopoulos, G. (2005). Improvement of xylose uptake and ethanol production in recombinant Saccharomyces cerevisiae through an inverse metabolic engineering approach. Appl. Environ. Microbiol. 71, 8249-8256.

Kambam, P.K.R., Sayut, D.J., Niu, Y., Eriksen, D.T., and Sun, L. (2008). Directed evolution of LuxI for enhanced OHHL production. Biotechnol. Bioeng. 101, 263-272.

Kambam, P.K.R., Eriksen, D.T., Lajoie, J., Sayut, D.J., and Sun, L. (2009). Altering the Substrate Specificity of RhlI by Directed Evolution. ChemBioChem 10, 553-558.

Khan, S., Brocklehurst, K.R., Jones, G.W., and Morby, A.P. (2002). The functional analysis of directed amino-acid alterations in ZntR from Escherichia coli. Biochem. Biophys. Res. Commun. 299, 438-445.

Kobayashi, H., Kærn, M., Araki, M., Chung, K., Gardner, T.S., Cantor, C.R., and Collins, J.J. (2004). Programmable cells: Interfacing natural and engineered gene networks. Proc. Natl. Acad. Sci. U. S. A. 101, 8414-8419. 
652 Lee, M.E., Aswani, A., Han, A.S., Tomlin, C.J., and Dueber, J.E. (2013). Expression-level 653 optimization of a multi-enzyme pathway in the absence of a high-throughput assay. $654 \quad$ Nucleic Acids Res. 41, 10668-10678.

655 Lu, J., Tang, J., Liu, Y., Zhu, X., Zhang, T., and Zhang, X. (2011). Combinatorial modulation of galP and glk gene expression for improved alternative glucose utilization. Appl. Microbiol. Biotechnol. 93, 2455-2462.

McGinness, K.E., Baker, T.A., and Sauer, R.T. (2006). Engineering controllable protein degradation. Mol. Cell 22, 701-707.

Michener, J.K., Thodey, K., Liang, J.C., and Smolke, C.D. (2012). Applications of geneticallyencoded biosensors for the construction and control of biosynthetic pathways. Metab. Eng. 14, 212-222.

Moon, T.S., Lou, C., Tamsir, A., Stanton, B.C., and Voigt, C.A. (2012). Genetic programs

Pitera, D.J., Paddon, C.J., Newman, J.D., and Keasling, J.D. (2007). Balancing a heterologous mevalonate pathway for improved isoprenoid production in Escherichia coli. Metab. Eng. 9, 193-207. engineering of Saccharomyces cerevisiae for the biotechnological production of succinic acid. Metab. Eng. 12, 518-525. 
Ro, D.-K., Paradise, E.M., Ouellet, M., Fisher, K.J., Newman, K.L., Ndungu, J.M., Ho, K.A., Eachus, R.A., Ham, T.S., Kirby, J., et al. (2006). Production of the antimalarial drug precursor artemisinic acid in engineered yeast. Nature 440, 940-943.

Rodrigues, A.L., Trachtmann, N., Becker, J., Lohanatha, A.F., Blotenberg, J., Bolten, C.J., Korneli, C., de Souza Lima, A.O., Porto, L.M., Sprenger, G.A., et al. (2013). Systems metabolic engineering of Escherichia coli for production of the antitumor drugs violacein and deoxyviolacein. Metab. Eng. 20, 29-41.

Rosen, R., Davidov, Y., LaRossa, R.A., and Belkin, S. (2000). Microbial sensors of ultraviolet radiation based on recA'::Lux fusions. Appl. Biochem. Biotechnol. - Part Enzyme Eng. Biotechnol. 89, 151-160.

Salis, H.M. (2011). Chapter two - The Ribosome Binding Site Calculator. In Methods in Enzymology, C. Voigt, ed. (Academic Press), pp. 19-42.

Salis, H.M., Mirsky, E.A., and Voigt, C.A. (2009). Automated design of synthetic ribosome binding sites to control protein expression. Nat. Biotechnol. 27, 946-U112.

Solomon, K.V., Sanders, T.M., and Prather, K.L.J. (2012). A dynamic metabolite valve for the control of central carbon metabolism. Metab. Eng. 14, 661-671.

Soma, Y., Tsuruno, K., Wada, M., Yokota, A., and Hanai, T. (2014). Metabolic flux redirection from a central metabolic pathway toward a synthetic pathway using a metabolic toggle switch. Metab. Eng. 23, 175-184.

Torella, J.P., Ford, T.J., Kim, S.N., Chen, A.M., Way, J.C., and Silver, P.A. (2013). Tailored fatty acid synthesis via dynamic control of fatty acid elongation. Proc. Natl. Acad. Sci. U. S. A. $110,11290-11295$.

Tsao, C.-Y., Hooshangi, S., Wu, H.-C., Valdes, J.J., and Bentley, W.E. (2010). Autonomous induction of recombinant proteins by minimally rewiring native quorum sensing regulon of E. coli. Metab. Eng. 12, 291-297.

Venayak, N., Anesiadis, N., Cluett, W.R., and Mahadevan, R. (2015). Engineering metabolism through dynamic control. Curr. Opin. Biotechnol. 34, 142-152.

Verma, N., and Singh, M. (2005). Biosensors for heavy metals. Biometals 18, 121-129.

Wang, J., Gong, Q., Maheshwari, N., Eisenstein, M., Arcila, M.L., Kosik, K.S., and Soh, H.T. (2014). Particle display: a quantitative screening method for generating high-affinity aptamers. Angew. Chem. Int. Ed Engl. 53, 4796-4801. 
713 Watstein, D.M., McNerney, M.P., and Styczynski, M.P. (2015). Precise metabolic engineering of carotenoid biosynthesis in Escherichia coli towards a low-cost biosensor. Metab. Eng., in review

Williams, T.C., Averesch, N.J.H., Winter, G., Plan, M.R., Vickers, C.E., Nielsen, L.K., and Krömer, J.O. (2015). Quorum-sensing linked RNA interference for dynamic metabolic pathway control in Saccharomyces cerevisiae. Metab. Eng. 29, 124-134.

Xu, P., Gu, Q., Wang, W., Wong, L., Bower, A.G.W., Collins, C.H., and Koffas, M.A.G. (2013). Modular optimization of multi-gene pathways for fatty acids production in E. coli. Nat. Commun. 4, 1409.

Xu, P., Wang, W., Li, L., Bhan, N., Zhang, F., and Koffas, M.A.G. (2014a). Design and Kinetic Analysis of a Hybrid Promoter-Regulator System for Malonyl-CoA Sensing in Escherichia coli. ACS Chem. Biol. 9, 451-458.

Xu, P., Li, L., Zhang, F., Stephanopoulos, G., and Koffas, M. (2014b). Improving fatty acids production by engineering dynamic pathway regulation and metabolic control. Proc. Natl. Acad. Sci. 111, 11299-11304.

Yadav, V.G., De Mey, M., Giaw Lim, C., Kumaran Ajikumar, P., and Stephanopoulos, G. (2012). The future of metabolic engineering and synthetic biology: Towards a systematic practice. Metab. Eng. 14, 233-241.

Yoon, S.-H., Lee, Y.-M., Kim, J.-E., Lee, S.-H., Lee, J.-H., Kim, J.-Y., Jung, K.-H., Shin, Y.-C., Keasling, J.D., and Kim, S.-W. (2006). Enhanced lycopene production in Escherichia coli engineered to synthesize isopentenyl diphosphate and dimethylallyl diphosphate from mevalonate. Biotechnol. Bioeng. 94, 1025-1032.

Zalatan, J.G., Lee, M.E., Almeida, R., Gilbert, L.A., Whitehead, E.H., La Russa, M., Tsai, J.C., Weissman, J.S., Dueber, J.E., Qi, L.S., et al. (2015). Engineering complex synthetic transcriptional programs with CRISPR RNA scaffolds. Cell 160, 339-350.

Zaslaver, A., Bren, A., Ronen, M., Itzkovitz, S., Kikoin, I., Shavit, S., Liebermeister, W., Surette, M.G., and Alon, U. (2006). A comprehensive library of fluorescent transcriptional reporters for Escherichia coli. Nat. Methods 3, 623-628.

Zhang, F., and Keasling, J. (2011). Biosensors and their applications in microbial metabolic engineering. Trends Microbiol. 19, 323-329. 
743 Zhang, F., Carothers, J.M., and Keasling, J.D. (2012). Design of a dynamic sensor-regulator

744 system for production of chemicals and fuels derived from fatty acids. Nat. Biotechnol.

$745 \quad 30,354-359$.

746 Zhao, J., Li, Q., Sun, T., Zhu, X., Xu, H., Tang, J., Zhang, X., and Ma, Y. (2013). Engineering

747 central metabolic modules of Escherichia coli for improving $\beta$-carotene production.

$748 \quad$ Metab. Eng. 17, 42-50.

749 